\title{
Creation and suppression of point defects through a kick-out substitution process of Fe in InP
}

\author{
Y. W. Zhao, ${ }^{\text {a) }}$ H. W. Dong, Y. H. Chen, Y. H. Zhang, J. H. Jiao, J. Q. Zhao, and L. Y. Lin \\ Materials Science Centre, Institute of Semiconductors, Chinese Academy of Sciences, P.O. Box 912, \\ Beijing 100083, People's Republic of China \\ S. Fung \\ Department of Physics, The University of Hong Kong, Hong Kong, People's Republic of China
}

(Received 31 January 2002; accepted for publication 6 March 2002)

\begin{abstract}
Indium antisite defect $\mathrm{In}_{\mathrm{P}}$-related photoluminescence has been observed in Fe-diffused semi-insulating (SI) InP. Compared to annealed undoped or Fe-predoped SI InP, there are fewer defects in SI InP obtained by long-duration, high-temperature Fe diffusion. The suppression of the formation of point defects in Fe-diffused SI InP can be explained in terms of the complete occupation by $\mathrm{Fe}$ at indium vacancy. The $\mathrm{In}_{\mathrm{P}}$ defect is enhanced by the indium interstitial that is caused by the kick out of In and the substitution at the indium site of Fe in the diffusion process. Through these Fe-diffusion results, the nature of the defects in annealed undoped SI InP is better understood. (C) 2002 American Institute of Physics. [DOI: 10.1063/1.1473695]
\end{abstract}

Impurity diffusion in semiconductors has been studied extensively in the past. In III-V compounds, the mechanism of atomic diffusion is well understood. ${ }^{1}$ Fast impurity diffusers, such as $\mathrm{Zn}$ and Fe, usually diffuse via the kick-out substitution mechanism in GaAs and $\mathrm{InP}^{2-5}$ In impurity diffused InP, defects such as indium precipitate, interstitial-type dislocation loops have been detected. ${ }^{6}$ Most of these studies on diffusion involve the comparison of the impurity profile with the fitting to the proposed model in which the influence of vacancy has been considered. ${ }^{3-5}$ However, the influence of impurity diffusion on the formation of point defects in diffused InP has rarely been reported.

In this letter, we present photoluminescence (PL) and photoinduced transient current spectroscopy (PITCS) results of semi-insulating (SI) InP obtained by high-temperature $\mathrm{Fe}$ diffusion. By comparing with the results of annealed undoped SI InP, it is found that a number of defects have been suppressed and an indium antisite $\mathrm{In}_{\mathrm{P}}$ defect is formed in Fe-diffused SI InP.

The samples used in the experiment were prepared by annealing $n$-type undoped InP wafers grown by the liquidencapsulated Czochralski (LEC) method. The electron concentration of as-grown LEC undoped InP was (3-6) $\times 10^{15} \mathrm{~cm}^{-3}$. The annealing was carried out in a sealed quartz tube at $930{ }^{\circ} \mathrm{C}$ for $80 \mathrm{~h}$. A quantity of $5 \mathrm{~N}$ Fe powder and $6 \mathrm{~N}$ red phosphorus at mole ratio of 1:2 were charged in the quartz tube before annealing. In this way, $\mathrm{FeP}_{2}$ with moderate vapor pressure can be formed at the annealing temperature $^{7,8}$ and $\mathrm{Fe}$ diffusion was thus realized. After annealing, the wafers were cooled at $40{ }^{\circ} \mathrm{C} / \mathrm{h}$ to room temperature. This process is found to convert the undoped $n$-type LEC InP to a SI material with resistivity and mobility above $10^{7} \Omega \mathrm{cm}$ and $3000 \mathrm{~cm}^{2} / \mathrm{V} \mathrm{s}$, respectively. The SI InP wafer so formed exhibits a good profile and radial uniformity. ${ }^{9}$ This technique has proved to be a promising method for the

a)Electronic mail: zhaoyw@red.semi.ac.cn preparation of high-quality SI substrates. It should be mentioned that undoped LEC InP with a carrier concentration of about $3 \times 10^{15} \mathrm{~cm}^{-3}$ can also be annealed into SI material under similar conditions, the only difference being the ambiance in the quartz tube is pure phosphorus. For PL and PITCS measurements, 0.6-mm-thick samples polished on one side were used. A layer of more than $60 \mu \mathrm{m}$ of the SI InP wafer was removed during the lapping and polishing process.

The PITCS system is a homemade setup with a conventional configuration. The PL system is a Bruck commercial ISF120 instrument equipped with an argon-ion laser and a germanium detector and the laser excitation power is $30 \mathrm{~mW}$.

PL spectra of Fe-diffused and undoped SI InP are shown in Fig. 1. A noticeable difference of the spectra is that a broad peak centered at $1.3 \mathrm{eV}$ can be observed in the Fediffused sample. The spectra of as-grown undoped $n$-type, Fe-doped and undoped SI InP are basically the same. Since the $1.3 \mathrm{eV}$ peak is only detected in Fe-diffused SI InP, it is reasonable to assume that this PL feature is associated with

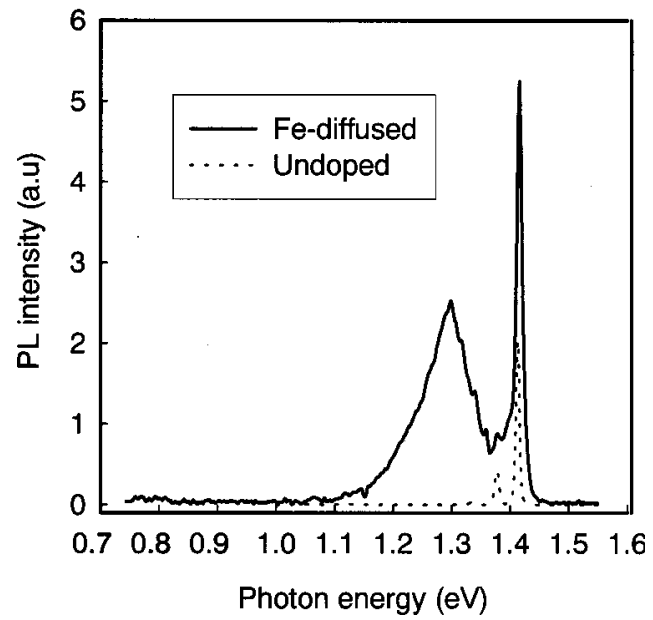

FIG. 1. Photoluminescence spectra at $10 \mathrm{~K}$ of Fe-diffused SI InP (solid line) and undoped SI InP (dashed line). 


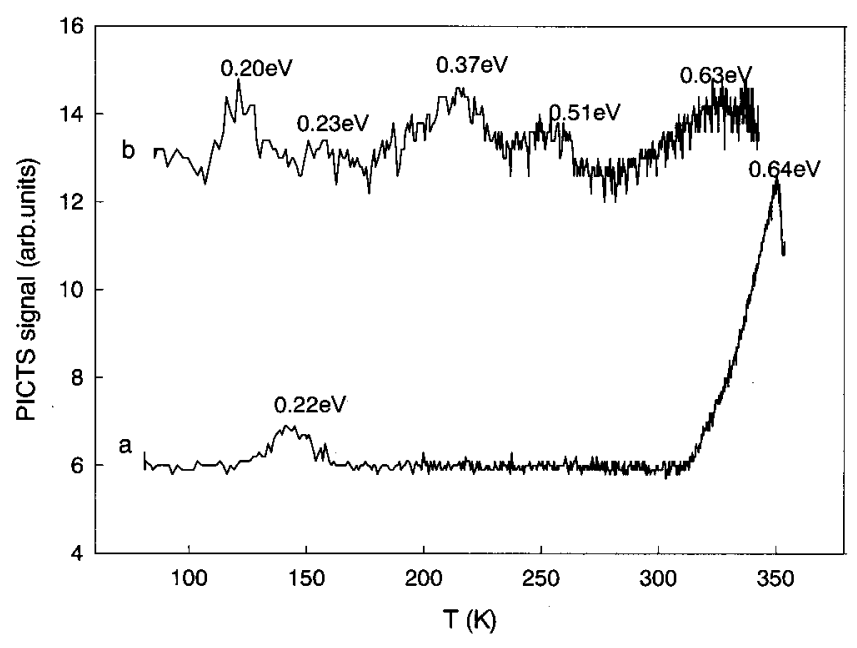

FIG. 2. Photoinduced transient current spectra of Fe-diffused SI InP (a) and undoped SI InP (b). Curves have been moved for better clarity.

Fe diffusion in the annealing process. A $1.3 \mathrm{eV}$ PL peak was also detected in electron-irradiated undoped and Fe-doped InP in the past. ${ }^{10-12}$ Experimental results have shown that this structure is due to the electron transition from the conduction band to the second ionized level of the indium antisite InP acceptor defect in InP. ${ }^{10,12}$ A PL peak at $1.393 \mathrm{eV}$, which corresponds to the transition of a conduction-band electron to the first ionized level of InP, ${ }^{12}$ can also be seen in the spectrum. Thus, our result seems to imply that indium antisite defect $\mathrm{In}_{\mathrm{P}}$ has been formed in Fe-diffused SI InP.

PITCS results of the Fe-diffused and undoped SI InP sample are shown in Fig. 2. Once again, an obvious difference can be seen between the two samples. Only two defects around 0.20 and $0.64 \mathrm{eV}$ have been observed in Fe-diffused SI InP. This is in great contrast to the five defects detected in the undoped SI InP material located at 0.22, 0.25, 0.37, 0.51, and $0.63 \mathrm{eV}$. Our results of defects in undoped SI InP are similar to those of Marrakchi et al. ${ }^{13}$ and Fang et al. ${ }^{14}$ It has also been found that the quantity and signature of defects in undoped SI InP are the same as those in SI InP prepared by annealing $n$-type InP predoped with a low concentration of Fe. ${ }^{13,15}$ Based on this analysis, it can be concluded that $\mathrm{Fe}$ diffusion has suppressed the formation of three defects in the SI InP material. It is interesting to note that nondiffusion $\mathrm{Fe}$ doping in InP does not produce such an effect, again indicating a correlation between Fe diffusion and defect suppression. As seen in Fig. 2, the Fe acceptor-related $0.64 \mathrm{eV}$ peak in Fe-diffused SI InP is much stronger. This fact suggests that Fe has substituted indium and acted as an effective deep compensation center in Fe-diffused SI InP.

A well-established difference between Fe diffusion and ordinary doping in InP is that the Fe impurity incorporated by doping during LEC growth in the latter process has already occupied an indium site before any subsequent annealing treatment. From our observation here, the formation of $\mathrm{In}_{\mathrm{P}}$ and the suppression of three defects in Fe-diffused SI InP seem most likely to be related to the diffusion mechanism. A model is invoked here to explain this observed phenomenon.

The mechanism of Fe diffusion in InP is believed to be a kick-out substitution process. ${ }^{2}$ Since Fe normally substitutes indium in InP, an indium interstitial will be created when a Downloaded 06 Nov 2006 to 147.8.21.97. Redistribution subject to
Fe diffuses in the material. During the subsequent hightemperature annealing process, the interstitial indium atom may have a strong tendency to occupy a phosphorus site, hence, forming an antisite defect in the Fe-diffused material. At the same time, as an additional effect, the substitutional occupation of $\mathrm{Fe}$ at the indium site means that indiumvacancy-related defects will also be greatly inhibited. A previous study has shown that the formation of these defects in undoped SI InP has a close relationship with the indium vacancy. ${ }^{16}$ Indeed, in as-grown InP a hydrogen vacancy complex can be detected. ${ }^{16,17}$ Experimental studies have confirmed that this complex is annihilated in annealed InP and an indium vacancy is produced as a result. ${ }^{16,18}$ Such a process can explain why there are more thermally induced defects in annealed undoped and Fe predoped SI InP than that in Fe-diffused SI InP. Our results also indicate that these defects are not impurity contamination related; otherwise, they cannot be suppressed by Fe diffusion. This interpretation is proving to be very helpful for the understanding of the nature of defects in annealed undoped SI InP.

The work described in this paper is partially supported by a grant from the Research Grant Council of the Hong Kong Special Administrative Region, China (under Project No. HKU7137/99P).

${ }^{1}$ B. Tuck, Atomic Diffusion in III-V Semiconductors (Hilger, Bristol, 1988).

${ }^{2}$ H. Zimmermann, U. Gösele, and T. Y. Tan, Appl. Phys. Lett. 62, 75 (1993).

${ }^{3}$ M. R. Brozel, E. J. Foulkes, and B. Tuck, Phys. Status Solidi A 72, K159 (1982).

${ }^{4}$ H. B. Serreze and H. S. Marek, Appl. Phys. Lett. 49, 210 (1986).

${ }^{5}$ S. Yu, T. Y. Tan, and U. Gösele, J. Appl. Phys. 69, 3547 (1991).

${ }^{6}$ D. Wittorf, A. Rucki, W. Jäger, R. H. Dixon, K. Urban, H. G. Hettwer, N. A. Stolwijk, and H. Mehrer, Appl. Phys. Lett. 77, 2843 (1995).

${ }^{7}$ D. Wolf, G. Hirt, and G. Müller, J. Electron. Mater. 24, 93 (1995).

${ }^{8}$ M. Uchida, T. Asahi, K. Kainosho, Y. Matsuda, and O. Oda, Jpn. J. Appl. Phys., Part 1 38, 985 (1999).

${ }^{9}$ Y. W. Zhao, H. W. Dong, J. H. Jiao, J. Q. Zhao, L. Y. Lin, N. F. Sun, and T. N. Sun, Chinese J. Semicond. 3, 285 (2002).

${ }^{10}$ F. P. Korshunov, S. I. Radautsan, N. A. Sobolev, I. M. Tiginyanu, V. V. Ursaki, and E. A. Kudryavtseva, Sov. Phys. Semicond. 23, 980 (1989).

${ }^{11}$ S. I. Radautsan, I. M. Tiginyanu, V. V. Ursaki, F. P. Korshunov, N. A. Sobolev, and E. A. Kudryavtseva, Solid State Commun. 7, 525 (1993).

${ }^{12}$ K. Kuriyama, K. Sakai, M. Okada, and K. Yokoyama, Phys. Rev. B 52, 14578 (1995).

${ }^{13}$ G. Marrakchi, K. Cherkaoui, A. Karoui, G. Hirt, and G. Müller, J. Appl. Phys. 79, 6947 (1996).

${ }^{14}$ Z. Q. Fang, D. C. Look, M. Uchida, K. Kainosho, and O. Oda, J. Electron. Mater. 27, L68 (1998).

${ }^{15}$ R. Fornari, A. Zappettini, E. Gombia, R. Mosca, K. Cherkaoui, and G. Marrakchi, J. Appl. Phys. 81, 7064 (1997).

${ }^{16}$ Y. W. Zhao, X. L. Xu, M. Gong, S. Fung, C. D. Beling, X. D. Chen, N. F. Sun, T. N. Sun, X. B. Guo, and Y. Z. Sun, Appl. Phys. Lett. 72, 2126 (1998).

${ }^{17}$ R. Darwich, B. Pajot, B. Rose, D. Robin, B. Theys, R. Rahbi, C. Porte, and F. Gendron, Phys. Rev. B 48, 17776 (1993).

${ }^{18}$ C. P. Ewels, S. Öberg, R. Jones, B. Pajot, and P. R. Briddon, Semicond.

Sci. Technol. 11, 502 (1996).
AIP license or copyright, see http://apl.aip.org/apl/copyright.jsp 